\title{
Management and Consideration of Natural Delivery in Pregnant Women with Suspected Coronavirus Pneumonia
}

\author{
Jing Li, Fuchuan Wang*, Yanhua Zhang, Li Li and Wei Yi \\ Department of Obstetrics and Gynecology, Capital Medical University, China
}

*Corresponding author: Fuchuan Wang, Department of Obstetrics and Gynecology, Beijing Di-Tan Hospital, Capital Medical University, China.

To Cite This Article: Jing Li, Fuchuan Wang, Yanhua Zhang, Li Li and Wei Yi. Management and Consideration of Natural Delivery in Pregnant Women with Suspected Coronavirus Pneumonia. A Review. Am J Biomed Sci \& Res. 2021 - 11(6). AJBSR.MS.ID.001685.

DOI: 10.34297/AJBSR.2021.11.001685.

Received: 眥 January 18, 2020; Published: 觜 February 04, 2021

\begin{abstract}
As of February 2020, a new type of coronavirus infection (coronavirus disease 2019, COVID-19) has continued to occur in China, and confirmed cases of infection in pregnant women have been reported. Beijing Ditan Hospital Affiliated with Capital Medical University is the designated hospital for treatment of maternal-neonatal coronavirus pneumonia in Beijing. Optimized management, prevention, and control of coronavirus pneumonia during delivery in pregnant women is crucial to ensuring the safety of mothers and infants. According to the latest COVID-19 national management plan combined with the specialization of our hospital in infectious diseases, we propose a process for management of birth in pregnant women with COVID-19 to provide clinical guidance and instruction for midwifery institutions.
\end{abstract}

Keywords: Coronavirus, Childbirth, Infection Prevention and Control, Pregnant Women, Midwifery, WHO, Pneumonia, National Health Commission, Transmission, Newborns, Suspected SARS-Cov-2

\section{Introduction}

Recently, the World Health Organization recognized a new coronavirus disease, coronavirus disease 2019 (COVID-19). The International Virus Classification Commission named the new coronavirus severe acute respiratory syndrome coronavirus 2 (SARS-CoV-2) [1]. Maternal cases of COVID-19 have occurred, with case reports of vertical transmission from mother to child. On February 12, 2020, Professor Huixia Yang, Professor Wei Hou, and Professor Yuanzhen Zhang and her team published an article in The Lancet Online analyzing the lack of evidence for vertical transmission in the uterus of pregnant women infected with SARSCoV-2. "Guidelines on the Management of Mothers and Newborns Infected with Pneumonia during the New Corona Virus Epidemic (First Edition)"

a. Opinion: Pregnant women with coronavirus pneumonia who are mildly ill and have good cervical conditions and can choose vaginal delivery [2]. Notice of the Beijing Municipal Commission of Health and Health on Printing and Distributing the Measures for Strengthening the Management of Coronavirus Infection in Pregnant Women in Beijing (First Edition) [3]. Beijing Ditan Hospital Affiliated with Capital Medical University is a designated hospital for pregnant women with confirmed COVID-19 pneumonia. These guidelines aim to effectively prevent and control COVID-19, ensure the safety of mothers and infants, and promote management during childbirth in conjunction with the National Health Commission's development and release of "Technical Guidelines for the Prevention and Control of New Types of Coronavirus Infection in Medical Institutions (First Edition) [4]" and other relevant protection guidelines. A case report of emergency delivery management of a pregnant women with suspected SARS-CoV-2 exposure. 
b. Keywords: suspected COVID-19, emergency delivery intervention, case report.

c. Case summary: A pregnant woman was 29 years old and $40+3$ weeks pregnant.

d. Anamnesis: In 2018, the patient denied a history of serious illness and had no history of surgery, trauma, drug allergy, or blood transfusion. Seven days prior, her husband returned from a high incidence area of COVID-19 and had COVID-19 symptoms. The pregnant woman appeared normal during pregnancy, without cough, sputum, chest tightness or other discomfort. She reported no fever before admission. A physical examination after admission showed a body temperature of $37.6^{\circ} \mathrm{C}$, pulse of 96 beats/min, Respiratory Rate (RR) of 20 breaths/min, blood pressure of $125 / 80 \mathrm{mmHg}$, and blood oxygen saturation of $96 \%$. Obstetric examinations were normal. Routine blood test results included a red blood cell count of $2.5 \times 1012 / \mathrm{L}$, white blood cell count of $2.4 \times 109 / \mathrm{L}$, platelet count of $30 \times 109 / \mathrm{L}$, hemoglobin level of $110 \mathrm{~g} / \mathrm{L}$, lymphocyte count of $0.9 \times 109 / \mathrm{L}$, and C-reactive protein level of $2.2 \mathrm{mg} / \mathrm{L}$. Her blood gas analysis, liver function, kidney function, D-dimer, and blood glucose levels were normal. The SARS-CoV-2 nucleic acid detection, influenza A virus nucleic acid detection, influenza B virus nucleic acid detection, influenza A H1N1 influenza virus RNA detection, and seasonal influenza virus $\mathrm{H} 3$ subtype nucleic acid detection results were not available.

e. Diagnosis: second pregnancy, 1 . The fetus was delivered at $40+3$ weeks of gestation with the headfirst, 2 . The membrane was ruptured, 3. SARS-CoV-2 was suspected.

f. Therapeutic intervention: As the pregnant woman neared delivery, she had a history of suspected SARS-CoV-2 exposure, accompanied by an increase in body temperature, and no SARSCoV-2 nucleic acid test results. The patient's epidemiological history and clinical symptoms were consistent with a diagnosis of the suspected COVID-19. She entered a negative pressure isolation delivery room through a dedicated channel to wait for delivery and to monitor her body temperature and delivery progress. On February 17, a live baby girl was delivered without complications with an uneventful delivery process. There was no abnormality after delivery, and the newborn was transferred to the isolation ward for observation. The patient received two negative SARSCoV-2 nucleic acid test results, and she was discharged normally 3 days after delivery. There were no abnormalities in the parturient and newborn, and they were followed up for 1 month after delivery.

Emergency medical protection measures included the implementation of three levels of protection for medical staff, and midwives and obstetricians entered the negative pressure isolation delivery room through the medical staff passage. All the test specimens from the pregnant woman are marked as "Suspected New Coronavirus Pneumonia", and the staff of the relevant departments were informed by telephone. This process helped implement the COVID-19 maternal delivery process in our hospital and further helped our hospital to improve relevant measures.

\section{Mother and Child Management During Childbirth}

\section{Establishment of a specialist infection ward - coronavirus maternity ward}

A pregnant woman diagnosed with COVID-19 or a newborn suspected or confirmed of having COVID-19 should be immediately admitted to a negative pressure isolation ward. The medical department and the nursing department should deploy manpower uniformly, and a specialist medical team consisting of an obstetrics specialist and members of the neonatal department should establish a specialist infection ward including a coronavirus ward for obstetrics and gynecology so that the obstetrics and neonatal departments can communicate effectively and in a timely manner. If high-risk prenatal cases occur, Multidisciplinary Team (MDT) management should be performed.

\section{Prenatal monitoring}

Studies have shown that although the number of infant infections is small, there is still a risk of SARS-CoV-2 infection [5]. Data show that pregnant women with viral pneumonia have premature delivery, restricted fetal growth and increased perinatal mortality [6]. In 2003, 7 newborns were delivered by pregnant women with SARS, including 2 cases of premature delivery and 3 cases of fetal distress [7], which deserve attention and reference. According to the "Expert Recommendations on Coronavirus Infection During Pregnancy and Puerperium", for pregnant women with COVID-19, electronic fetal heart rate monitoring and ultrasonography are recommended to evaluate the condition of the fetus based on the gestational week [8]. Obstetricians, infectious disease surgeons, pediatricians, and ultrasound specialists should jointly determine the delivery method. After full-term pregnancy, fetal heart rate monitoring should be performed every 2 hours, and the results should be consistent with type I fetal monitoring or recoverable type II fetal heart rate monitoring. Continuous ultrasonography should be used to assess fetal structure, growth, and development. If necessary, maternal consent should be obtained, and magnetic resonance examination and prenatal diagnostic screening can be performed.

\section{Delivery management}

Management of isolation and the negative pressure delivery room: A pregnant woman with COVID-19 who is about to give birth (first birth, dilation of $10 \mathrm{~cm}$; second and multiple births, dilation of $3 \mathrm{~cm}$ ) should enter the negative pressure delivery 
room. The negative pressure delivery room should be intact, meet the "Environmental Control Requirements for Hospital Negative Pressure Isolation Ward" (GBT 35428-2017) [9] and be in a standby state. Special items such as masks, caps, protective screens, and isolation clothing should be provided at the door of the negative pressure delivery room. Equipment such as fetal heart monitors and neonatal radiation tables should be present in the negative pressure delivery room. Other than instruments and equipment, deliveries should use single-use products.

Management of the receiving process: One obstetrician and pediatrician and two midwives should be called $10 \mathrm{~min}$ in advance and enter the delivery room, and three levels of protection should be provided by the entrance for the medical staff. The infectious disease department and respiratory department should be always on standby. According to the "Notice on Printing and Distributing Guidance for Protecting Medical Staff from Coronavirus Pneumonia Infection in Beijing " issued on February 3, 2020, the exposure classification of childbirth is a high risk. Protective equipment, such as protective medical clothing (disposable), isolation clothing, protective medical masks, work caps, protective screens/goggles, double gloves, and shoe covers/boot covers, should be used [10]. After the pregnant woman (who always wears an N95 protective mask) enters the negative pressure delivery room, $2000 \mathrm{mg} / \mathrm{L}$ chlorine-containing disinfectant should be used to sterilize the area where she passed through. Medical staff should enter the negative pressure delivery room and change positions every 4 hours to reduce staff turnover and conserve protective materials. To obtain items during delivery and for the entry of electronic medical record information, radios should be used to communicate with workers outside the negative pressure delivery room.

Delivery management: Close observation of the labor process, monitoring of the vital signs, blood oxygen saturation, subjective symptoms, cardiopulmonary condition, and continuous low-flow oxygen inhalation of the patient and fetal heart monitoring are recommended. Continuous process Electrocardiogram (ECG) monitoring and pulse oxygen monitoring are recommended to avoid hypoxia and heart rhythm abnormalities. As the length of labor extends, the contractions will become increasingly stronger, oxygen consumption is increased, and gas exchange in the lungs becomes insufficient. Therefore, signs of intrauterine fetal distress could occur. The second stage of labor involves excessive exertion, breath-holding and abdominal pressure, and the process should be shortened as much as possible. The standard "New Coronavirus Pneumonia Diagnosis and Treatment Plan (Trial Version 6)"[11] criteria for severe cases include shortness of breath $(R R \geq 30$ breaths/min); resting status, oxygen saturation $\leq 93 \%$; and arterial oxygen pressure (PaO2)/oxygen concentration (FiO2) $\leq 300 \mathrm{mmHg}$. In severe cases, a switch to a cesarean section should be performed as soon as possible, and the pregnancy should be terminated.
Newborn management: Delayed ligation of the umbilical cord is not performed after delivery of the newborn, and the amniotic fluid and blood stains of the newborn should be cleaned as much as possible. Newborn infants should be given a preliminary physical examination by a neonatal physician and vaccinated in accordance with the specifications. The pediatrician should determine whether abnormalities are present and then use an infant incubator to transfers the newborn to the coronavirus ward of the obstetrics and gynecology department through a dedicated elevator. The isolation observation/diagnosis period should be more than 14 days. During the quarantine period, the newborn should be provided with complete monitoring and treatment, and donated breast milk can be used to avoid breastfeeding [9]. It is recommended to pump milk regularly to ensure lactation, and breastfeeding is not feasible until SARS-CoV-2 infection is ruled out or cured [12].

Placenta and medical device management: The placenta of a pregnant woman with COVID-19 should be treated as infectious disease waste. The treated placenta should be double wrapped in a yellow garbage bag, an infectious waste label indicating "COVID-19" should be affixed, and the bag should be collected by a specific person. Medical equipment should include disposable items. Reusable medical devices should be immersed in a $2000 \mathrm{mg} / \mathrm{L}$ chlorine disinfectant for $30 \mathrm{~min}$. After replacing their gloves, the midwife should remove the instruments, rinse them with water, put them into a double yellow garbage bag, put an infectious disease label of "COVID-19" on the bag, put the bag in the designated place, and notify the supply disinfection center to register for recycling. The medical instruments should be disinfected and wiped twice a day (it is best to use disposable disinfection wipes) to ensure that they are in a standby state.

Maternal psychological management: The occurrence of pneumonia diagnosed as SARS-CoV-2 infection is a considerable source of stress, which causes uncertainty and insecurity. Coupled with natural delivery, patients may experience anxiety, such as a stress response, and even symptoms of depression. According to the "Guiding Principles for Emergency Psychological Crisis Intervention for Pneumonia Epidemic of Coronavirus Infection" [13], midwives need to fully inform the pregnant women of their disease information during childbirth and the special treatment that will be provided in infant wards. During the delivery process, the meaning of self-protection and isolation should be explained to the patient to create a sense of security, and the use of nondrug analgesics such as music can help reduce the pain and fear of childbirth. Obstetricians, pediatricians, and midwives should communicate with pregnant women in a timely manner, repeatedly informing them objectively of childbirth safety information. Patients should be informed that negative psychological reactions will damage the normal immune system of the human body and are not conducive to childbirth. Particular attention and continuous follow-up are needed to 
determine whether postpartum depression is present, which is one of the most associated emotional disorders in perinatal women, with a global incidence of 17.7[14]. During the epidemic, pregnant women should not only take good care of themselves but also pay attention to their mental health. If necessary, they can also conduct self-monitoring and screening through the Edinburgh Postpartum Depression Screening Scale. They should limit their exposure to epidemic information and educate family members and patients [15].

\section{Further Consideration of Related Issues in Midwifery}

\section{Effective communication with pregnant women during the delivery process}

During childbirth, the midwife should provide overall and multifaceted support to the mother and explain information regarding childbirth, including the natural process of childbirth, exertion techniques, appropriate breathing methods, how to divert pain, distraction, and anxiety. However, pregnant women with COVID-19 need to wear N95 protective masks during labor, and midwives should have tertiary protection, which can easily cause poor communication, such as poor hearing and misunderstanding. It is worth exploring how to effectively communicate with pregnant women during the delivery process. Body language mainly refers to the use of body movements instead of language for the purpose of communication [16]. Practitioners should confirm issues and communicate with pregnant women in advance, and specific meanings can be expressed by changing the frequency of tapping on a pregnant women's legs. For example, continuous tapping of the legs means continuous exertion. Two taps mean pause and rest.

\section{Reducing occupational exposure during the delivery process.}

Three levels of protection require two layers of gloves and two layers of gloves in the staging area (surgical hand cleaning cannot be performed). A total of four layers of gloves results in poor finger flexibility and sensitivity. In the second stage of labor, when the fetal head is exposed after the perineum tightens, the midwife should control the speed of the fetal head with one hand and hold the perineum with the other hand. If a perineal incision is required, actions such as perineal tissue anesthesia, side incision, and suturing should be performed (which require more flexibility and sensitivity of the hands). For such patients, the expert guidance is to shorten the labor process and implement lateral perineal resection [17]. Further confirmation is required to determine whether perineal incisions and sutures increase the risk of occupational exposure of midwives in the case of poor finger flexibility and sensitivity. The author suggests that most pregnant women with COVID-19 who undergo childbirth are patients with less severe disease. During the delivery process, comprehensive consideration should be given to minimize the degree of perineal injury, reduce the difficulty of suturing, and increase the suture speed instead of performing conventional lateral perineal resection [18].

\section{Summary}

During the outbreak of coronavirus-associated pneumonia, pregnant women are a special population, and protection against the epidemic is as important as maternal and child health. Using the latest diagnosis and treatment plan and effective resources, a good natural environment should be created to the greatest extent possible. Protecting the health of mothers and infants while reducing cross-infection and occupational exposure to health care workers requires medical staff members to study and explore different options together.

\section{References}

1. Huijun Chen, Juanjuan Guo, Chen Wang, Fan Luo, Xuechen Yu, et al. (2020) Clinical characteristics and intrauterine vertical transmission potential of COVID-19 infection in nine pregnant women: a retrospective review of medical records. Lancet 395(10226): 809-81.

2. (2020) Expert Group of Union Hospital of Tongji Medical College, Huazhong University of Science and Technology, Guiding Opinions on Management of Maternal and Newborns during the Epidemic of Pneumonia of New Coronavirus (First Edition) [J]. Tongji Hospital of Huazhong University of Science and Technology.

3. (2020) Beijing Municipal Health Commission. Notice on Issuing the Measures for Strengthening the Administration of Pregnancy Combined with New Coronavirus Infection in Beijing (First Edition) [Z]

4. (2020) General Office of the National Health Commission. Technical Guidelines for the Prevention and Control of New Coronavirus Infections in Medical Institutions (First Edition) [Z].

5. Min Wei, Jingping Yuan, Yu Liu, Tao Fu, Xue Yu, et al, (2020) Novel Coronavirus Infection in Hospitalized Infants Under 1 Year of Age in China. JAMA 323(13): 1313-1314.

6. Chen YH, Keller J, Wang IT, Ching C, Herng C, (2012) Pneumonia and pregnancy outcomes: a nationwide population-based study[J]. Am J Obstet Gynecol 207(4): 288.

7. Wang Yunhui, Zhang Rui, Zhang Jianping, et al. (2004) Impact of SARS infection on pregnancy outcome and fetus and infant [J]. Chinese Journal of Perinatal Medicine 7 (3): 155-158.

8. (2020) Professional Committee of Maternal and Fetal Physicians of the Chinese Physician Association of Obstetricians and Gynecologists, Editorial Board of Chinese Journal of Perinatal Medicine. Expert advice on coronavirus infection during pregnancy and puerperium [J]. Chinese Journal of Perinatal Medicine 23(00).

9. GB / T 35428-2017, Environmental Control Requirements for Hospital Negative Pressure Isolation Ward[S].

10.(2020) Notice on Printing and Distributing Guidance for Protecting Pneumonia Medical Staff of Coronavirus Infection in Beijing [S]. Beijing: Commission for Health and Health.

11. (2020) Notice on Printing and Distributing the New Corona Virus Pneumonia Diagnosis and Treatment Plan (Trial Version 6) [S]. National Health Office Medical Letter No.145.

12. Schwartz DA (2020) An analysis of 38 pregnant women with COVID-19, their newborn infants, and maternal-fetal transmission of SARS-CoV-2: maternal coronavirus infections and pregnancy outcomes. Arch Pathol Lab Med. 
13. Zhongguo Dang (2020) [Perinatal and neonatal management plan for prevention and control of 2019 novel coronavirus infection (1st Edition)]. China Journal of Contemporary Pediatrics 22(2): 87-90.

14. (2020) Guidelines for emergency psychological crisis intervention for pneumonia epidemic of Coronavirus infection [S]. Nation: Commission for Health and Health.

15. Song Peige, Yu Jinyue, Chan Kit Yee, Evropi T, Igor Rudan, (2018) Prevalence, risk factors and burden of diabetic retinopathy in China: a systematic review and meta-analysis[J]. J Glob Health 8(1): 010803.
16. Ma Liangkun, Duan Yanping, Zheng Ruimin, Zeng Xuejun, Liu Juntao, et al. (2020) Expert advice on maternal psychological adjustment during the prevention and control of coronavirus pneumonia epidemic [J]. Xiehe Medical Journal.

17. Wang Xijuan. Analysis on the application of Body Language in Vocal Music Performance [J]. Music Space-Time 504 (1): 111-128.

18. Liu Xinghui, He Jing, Qi Hongbo (2018) Midwifery [M]. Beijing: People’s Medical Publishing House. 276-278. 